\title{
Outcomes of Critical Limb Ischemia in Hemodialysis Patients After Distal Bypass Surgery - Poor Limb Prognosis With Stage 4 Wound, Ischemia, and Foot Infection (WIfI) -
}

Katsuyuki Hoshina, PhD; Kota Yamamoto, PhD; Tetsuro Miyata, PhD; Toshiaki Watanabe, PhD

\begin{abstract}
Background: Distal bypass is the first-line treatment for patients with critical limb ischemia (CLI). In Japanese highvolume centers, approximately half of these patients are on hemodialysis (HD). We have treated such patients first with bypass using a multidisciplinary perioperative strategy. We reveal the recent characteristics of patients who underwent distal bypass and the surgical outcomes in Japan, especially focusing on the foot conditions by using the wound, ischemia, and foot infection (WIfl) classification.
\end{abstract}

Methods and Results: The 152 patients underwent distal bypass in a tertiary center hospital, and we compared patients on HD (HD group) to those not on HD (non-HD group). There were significant differences between the 2 groups in the overall survival, major adverse cardiac event-free survival and amputation-free survival (AFS) rates $(P<0.0001)$. The procedural outcomes were analyzed via primary and secondary patency, and there was no difference. In the subanalysis of limb status using WIfl stage, the AFS rate of the HD group was significantly worse than that of the non-HD group for WIfl stage 4 patients.

Conclusions: The life and limb prognoses of patients with CLI and HD were worse than those of non-HD patients. There was no difference in surgical outcomes suggested by the graft patency rates between the 2 groups. AFS in WIfl stage 4 was significantly worse in the HD group, which indicated the importance of preoperative limb status. (Circ J 2016; 80: 2382-2387)

Key Words: Distal bypass; Hemodialysis; Japanese; Wound, ischemia, and foot infection (WIfl) classification

D istal bypass is a good treatment option for improving the symptoms of critical limb ischemia (CLI), and it is the first line of treatment in the endovascular era. ${ }^{1-3}$ If arterial reconstruction is successfully performed, major limb amputation can be avoided in many CLI patients, which subsequently helps them maintain their quality of life. However, vascular surgeons must consider each CLI patient's prognosis from 2 points of view: limb and life prognoses. In order to perform the bypass procedure successfully, we have to check the vessel for conditions such as calcification of the artery, peripheral vessel run-off, and the diameter of the candidate conduit, and we also have to plan how to access the anastomotic site and perform bypass routing. The bypass patency should be dependent on this perioperative planning. At the same time, we should also consider the life expectancy of CLI patients, because their comorbidities, including coronary risks, are severe. ${ }^{4}$ This is supported by the fact that the survival rate of CLI patients was revealed to be equivalent to that for some types of advanced cancers. 5

The number of patients who undergo hemodialysis (HD) has drastically increased in Japan, which might be strongly correlated to the increase in diabetes mellitus (DM). ${ }^{6}$ CLI patients with HD and DM might be admitted to high-volume centers, at which most of the preoperative risk evaluation and foot care are systematically structured. In Japan, approximately half of CLI patients in these high-volume centers are also on HD (Figure 1). ${ }^{7-10}$ Despite a relatively large population of high-risk patients, the outcomes of distal bypass in CLI patients have been acceptable; limb salvage rate and survival rate at 5 years after surgery of approximately $80 \%$ and $60 \%$, respectively. ${ }^{7}$

We have previously reported the prognosis of CLI patients who underwent distal bypass up to $2010 .^{7,11}$ The foot and systemic conditions of CLI patients who are admitted to our

Received June 16, 2016; revised manuscript received September 6, 2016; accepted September 12, 2016; released online October 4, 2016 Time for primary review: 39 days

Division of Vascular Surgery, Department of Surgery, Graduate School of Medicine, The University of Tokyo, Tokyo (K.H., K.Y., T.W.); Sanno Medical Center, International University of Health and Welfare, Tokyo (T.M.), Japan

Mailing address: Katsuyuki Hoshina, MD, Division of Vascular Surgery, Department of Surgery, Graduate School of Medicine, The University of Tokyo, 7-3-1 Hongo, Bunkyo-ku, Tokyo 113-8655, Japan. E-mail: traruba@ gmail.com

ISSN-1346-9843 doi:10.1253/circj.CJ-16-0591

All rights are reserved to the Japanese Circulation Society. For permissions, please e-mail: cj@j-circ.or.jp 


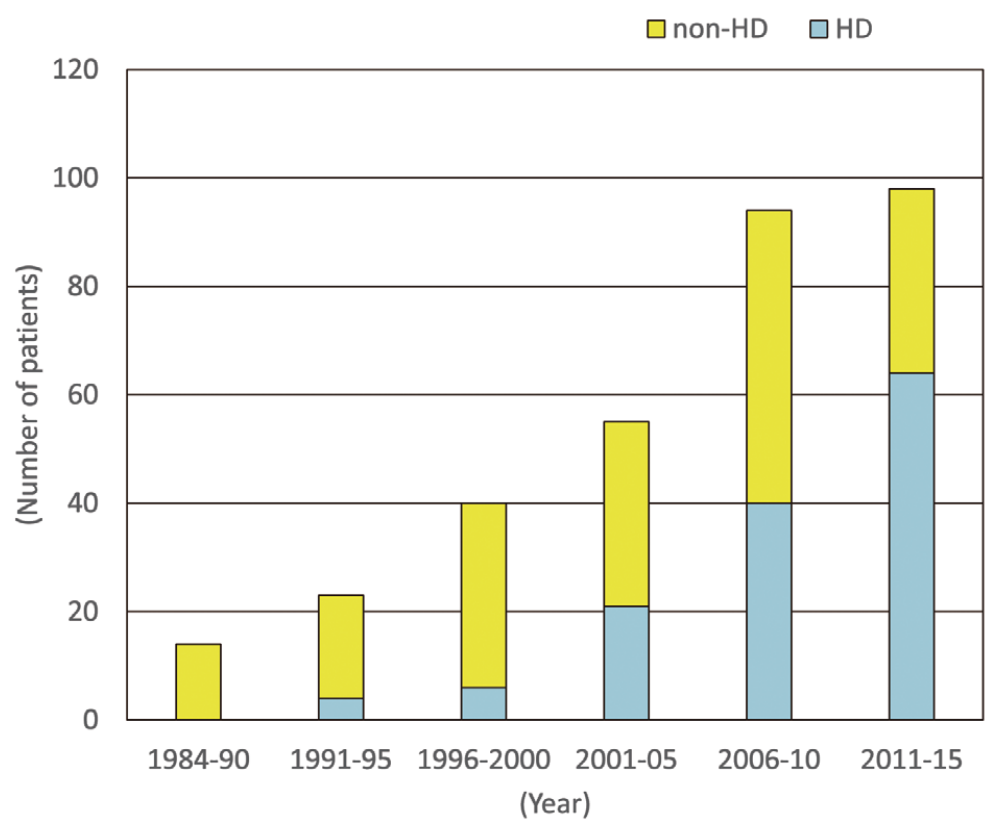

Figure 1. Rate of hemodialysis (HD) in patients undergoing distal bypass surgery for critical limb ischemia in Japan.

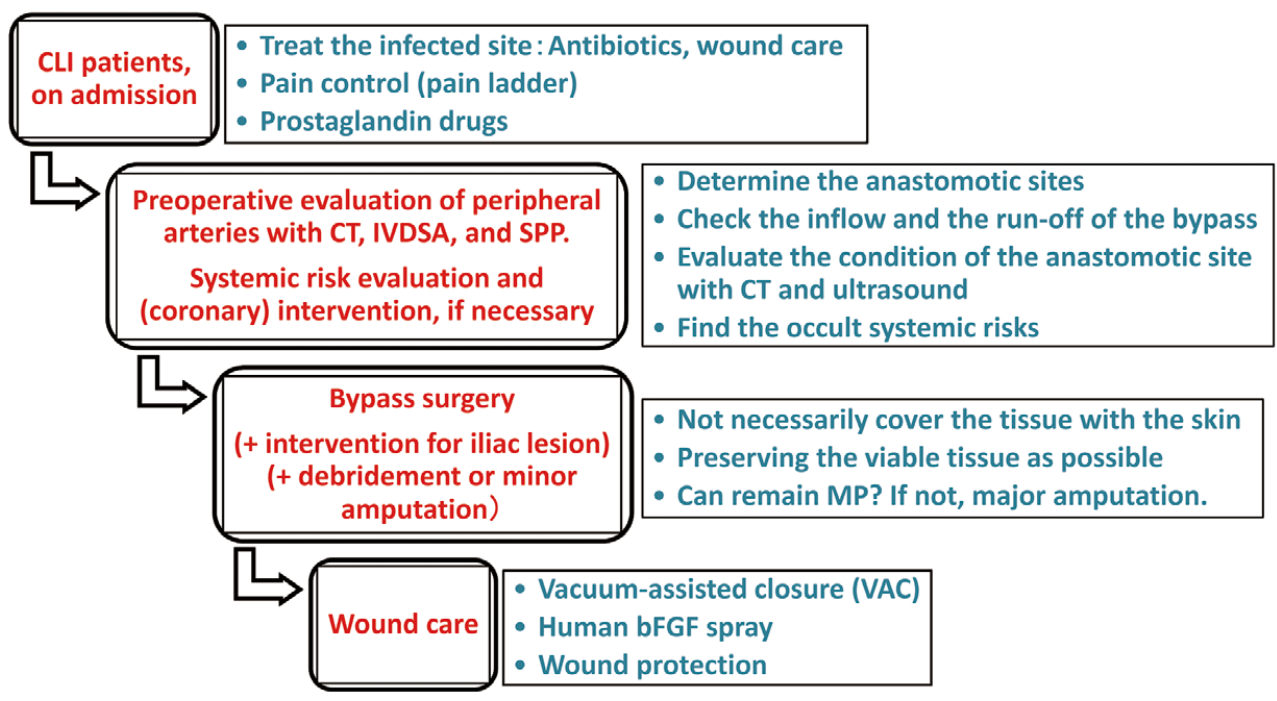

Figure 2. Institutional perioperative strategy for critical limb ischemia (CLI) patients. bFGF, human basic fibroblast growth factor; CT, computed tomography; IVDSA, intravenous digital subtraction angiography; MP, metatarsophalangeal joint; SPP, skin perfusion pressure.

specific tertiary high-volume center have tended to worsen, possibly affected by the increase of DM, so we considered it necessary to perform further evaluation.

In this study, we wanted to elucidate the recent outcomes of HD patients after distal bypass for CLI under multidisciplinary treatment in a tertiary high-volume center, from the life and the limb prognostic point of view. Next, as limb condition varies widely, especially in HD patients, we evaluated it preoperatively using a risk stratification based on the wound, ischemia, and foot infection (WIfI) classification ${ }^{12}$ and analyzed the limb outcomes per stage. We hypothesized that WIfI staging might reveal the characteristic features of this study population, especially of the HD group, and influence the operative strategy of distal bypass.

\section{Methods}

This study was conducted according to the guidelines of the research ethical committee of the University of Tokyo. We retrospectively reviewed 152 patients with CLI who were admitted to hospital and underwent distal bypass between January 2005 and December 2014. We divided these patients 


\begin{tabular}{|c|c|c|c|}
\hline Variable & HD group & Non-HD group & $P$ value \\
\hline $\mathrm{n}$ & 73 & 79 & \\
\hline Age & $66.7 \pm 10.1$ & $70.6 \pm 13.5$ & 0.044 \\
\hline Male sex & $55(75 \%)$ & $50(63 \%)$ & 0.12 \\
\hline \multicolumn{4}{|l|}{ Comorbidities } \\
\hline Hypertension & $57(78 \%)$ & $56(71 \%)$ & 0.36 \\
\hline Ischemic heart disease & $32(44 \%)$ & $22(28 \%)$ & 0.044 \\
\hline Cerebrovascular disease & $23(32 \%)$ & $19(24 \%)$ & 0.37 \\
\hline Diabetes mellitus & $59(81 \%)$ & $37(47 \%)$ & $<0.0001$ \\
\hline Dyslipidemia & $19(26 \%)$ & $23(29 \%)$ & 0.72 \\
\hline \multicolumn{4}{|l|}{ Symptoms } \\
\hline Rest pain & $9(12 \%)$ & $16(20 \%)$ & \multirow{3}{*}{0.023} \\
\hline Ulcer & $24(33 \%)$ & 37 (47\%) & \\
\hline Gangrene & $40(55 \%)$ & $26(33 \%)$ & \\
\hline $\mathrm{n}$ & 71 & 74 & \\
\hline \multicolumn{4}{|l|}{ WIfl classification } \\
\hline 1 & $9(13 \%)$ & $3(4 \%)$ & \multirow{4}{*}{0.17} \\
\hline 2 & $18(25 \%)$ & 25 (34\%) & \\
\hline 3 & $21(30 \%)$ & $26(35 \%)$ & \\
\hline 4 & $23(32 \%)$ & $20(27 \%)$ & \\
\hline
\end{tabular}

WIfl; wound, ischemia, and foot infection.

into 2 groups: 73 patients who were on HD (HD group) and 79 patients who were not (non-HD group).

\section{Preoperative Management}

The details of preoperative management have been described previously. ${ }^{7,13}$ On admission of CLI patients, we controlled their chronic ischemic pain with nonsteroidal anti-inflammatory drugs and opioid drugs, and administrated antibiotics prophylactically for infection in the tissue loss site. We routinely referred patients to the comfort care team and infection survey team of the hospital. Prostaglandin E1 (Alprostadil ${ }^{\circledR}$ ) was sometimes used. The preoperative routine study included cardiopulmonary, gastrointestinal, and carotid artery diseases and other comorbidities, and all patients underwent consultation with an anesthesiologist. Distal bypass surgery was finally decided with consideration of the patient's risks (Figure 2).

\section{Patients' Characteristics}

Patients' characteristics were analyzed for each group (HD vs. non-HD group), including age, sex, hypertension, DM, dyslipidemia, ischemic heart disease (IHD), and cerebrovascular disease (CVD). IHD was defined as documented definite angina or myocardial infarction, or $>50 \%$ stenosis of at least 1 coronary artery. CVD was defined as documented prior stroke or transient ischemic attack (Table).

\section{Wound Evaluation and Bypass Indication}

As we described previously, the severity of ischemia was assessed using the ankle-brachial pressure index and skin perfusion pressure (SPP). Besides classical classification of peripheral arterial disease, such as Fontaine or Rutherford, we included the SPP, which is reported as a predictor of wound healing, in the diagnostic criteria of CLI. In addition, we evaluated the CLI status by referring to the WIfI classification. $^{12}$ We retrospectively reviewed the electronic database and classified the status using images of the patients' feet taken by the foot care team. As the SPP is accepted widely in Japan, we valued the ischemic parameter of WIfI as a substi- tute of toe pressure. ${ }^{14,15} \mathrm{We}$ determined the distal anastomotic site with both a subtraction image from intra-arterial angiography and ultrasonography. For more accurate identification of the appropriate anastomotic site, we used an exploratory image overlay system with a tablet PC for 10 CLI patients. ${ }^{16}$

\section{Surgical Procedures}

Concomitant proximal artery lesions in the aortoiliac and femoropopliteal arteries were simultaneously revascularized via bypass surgery or endovascular treatment. For CLI patients, we aimed at complete revascularization involving crural or foot artery lesions as much as possible using distal bypass surgery. We used the auto-vein with a diameter $>2.5 \mathrm{~mm}$ for a conduit. Artificial grafts were not used. The non-dissection method was our way of clamping the vessels, using 250$400 \mathrm{mmHg}$ of air tourniquet pressure. As HD patients tend to have severely calcified arteries, we prefer this method over direct arterial clamping. Infectious or gangrenous tissues were resected at the time of revascularization and remained uncovered with skin.

\section{Postoperative Wound Care}

As the outcomes of uncovered wounds directly affect the limb prognosis, we checked the wound daily, with additional debridement if necessary, and performed several treatments: local administration of recombinant human basic fibroblast growth factor (Fiblast ${ }^{\circledR}$; Kaken Pharmaceutical Co, Ltd, Tokyo, Japan), prostaglandin ointment, vacuum-assisted closure therapy (VAC ${ }^{\circledR}$; Kinetic Concepts, San Antonio, TX, USA), and skin graft or free flap transfer. Patients were discharged from the hospital after confirmation of the wound's stability.

\section{Postoperative Surveillance and Outcome Assessment}

The patients were surveyed with routine physical examinations, wound care, and ultrasonography for graft flow every 3-6 months in the first 2 years after the surgery. Primary and secondary graft patency, the limb salvage rate, survival rate, amputation-free survival (AFS), and major adverse cardiovas- 
A

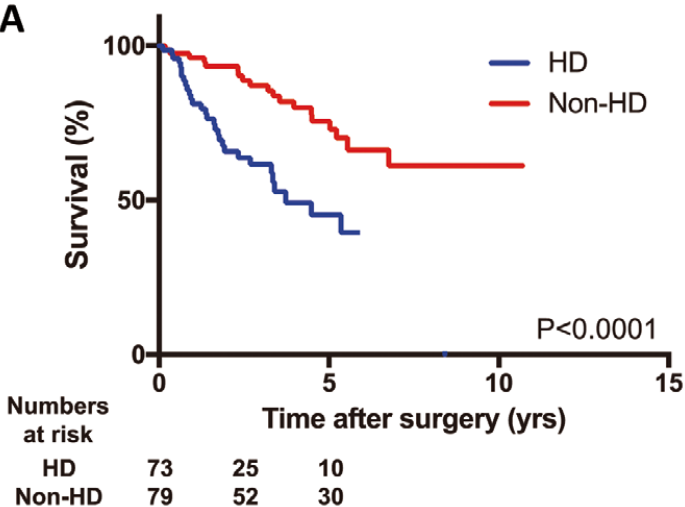

B

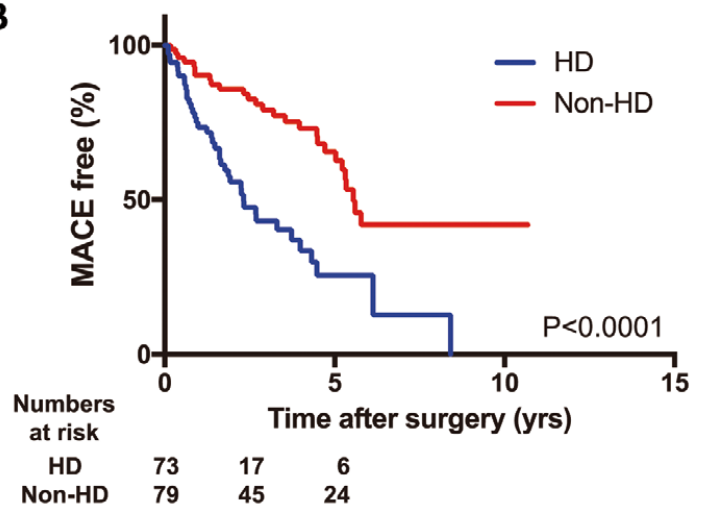

Figure 3. Long-term outcomes linked to life prognosis after distal bypass for critical limb ischemia: HD group vs. non-HD group. (A) Overall survival rate, (B) MACE-free survival rate. HD, hemodialysis; MACE, major adverse cardiac events.

cular events (MACE) were analyzed. We focused on the clinical stage of the WIfI classification, on the assumption that the stage would accurately reflect the limb prognosis. In some settings of combined clinical stages, we compared the HD group to the non-HD group.

\section{Statistical Analysis}

Statistical analysis was performed with JMP ${ }^{\circledR} 9.0$ software (SAS Institute Inc, Cary, NC, USA). Continuous data are expressed as mean \pm standard deviation. Univariate analyses were conducted with Student's t-test for continuous data and chi-square test for categorical data. Long-term outcomes were assessed using the Kaplan-Meier method with the log-rank test. A $\mathrm{P}$-value $<0.05$ was considered significant for each analysis.

\section{Results}

\section{Characteristics of the HD Group}

Compared with the non-HD group, the patients in the HD group were younger and were more likely to have IHD $(\mathrm{P}=0.044)$ and $\mathrm{DM}(\mathrm{P}<0.0001)$. Regarding the limb status, the HD group consisted of more patients with gangrene $(\mathrm{P}=0.023)$, but there was no difference in WIfI stage between the 2 groups $(\mathrm{P}=0.17)$ (Table).

\section{Long-Term Outcomes}

We evaluated the life prognosis by comparing the survival rates (overall and MACE-free) of the 2 groups. There were significant differences in these survival rates $(\mathrm{P}<0.0001)$ (Figure 3). The limb prognosis by AFS was worse in the HD group $(\mathrm{P}=0.0004)$ (Figure 4).

The surgical outcomes were analyzed via primary and secondary patency, and we could not find differences between the 2 groups. P-values were 0.57 for primary patency and 0.11 for secondary patency, respectively (Figure 5).

The observation period was $3.4 \pm 2.4$ years. Within this period, death, MACE, graft failure, and major amputation occurred in $51,69,20$ and 16 patients, respectively. In order to exclude the bias of limb status between the 2 groups as much as possible, we focused on the limb prognosis by comparing the AFS rate per WIfI stage between groups (Figure 6). As the number of patients in stages 1 and 2 was small, we analyzed the data for stages 1-3 and for stage 4 . In this series, the AFS rate in the

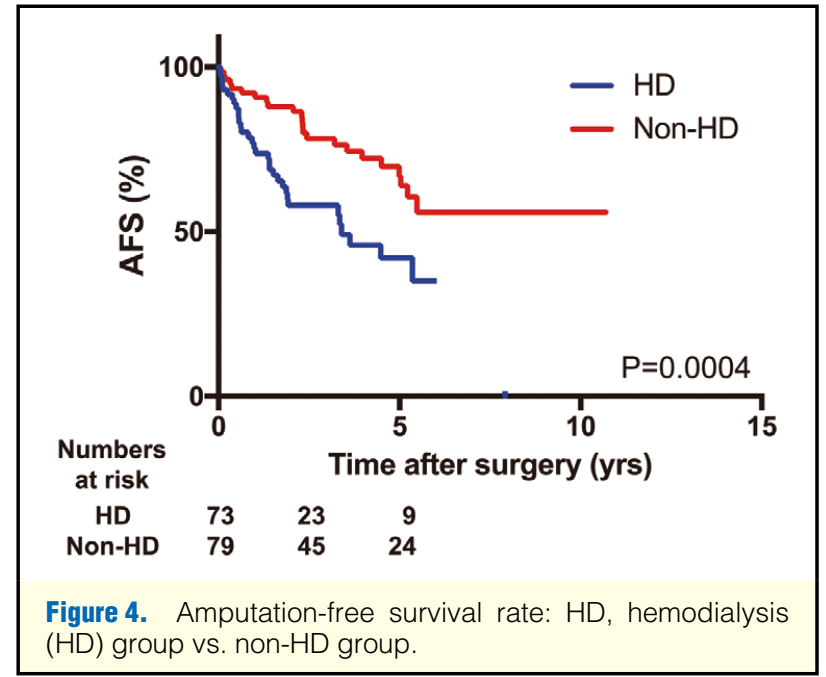

HD group tended to be worse than that in the non-HD group in stages $1-3(\mathrm{P}=0.06)$, and was significantly worse in stage 4 $(\mathrm{P}=0.0016)$.

\section{Discussion}

Although we compared the HD group with the non-HD group in the present study, it is not necessarily accurate to link this single parameter of HD to the outcome of distal bypass surgery. There are many confounders in the atherosclerotic pathogenesis and the pathway to CLI. Among them, the most critical confounder was DM, as shown in the Table. However, even after adjusting for other parameters such as DM, dyslipidemia, and hypertension, HD was still revealed to be an independent risk for cardiovascular events. ${ }^{17}$ When performing distal bypass for HD patients, we sometimes found that severe arterial calcification made the procedure more difficult. One of the scenarios of calcification is that elevated levels of phosphorus might play an important role in vascular calcification by transforming vascular smooth muscle cells into osteoblast-like cells. ${ }^{18-20}$ Considering the pathophysiological state of uremic calcification and accompanying technical difficulty 
A

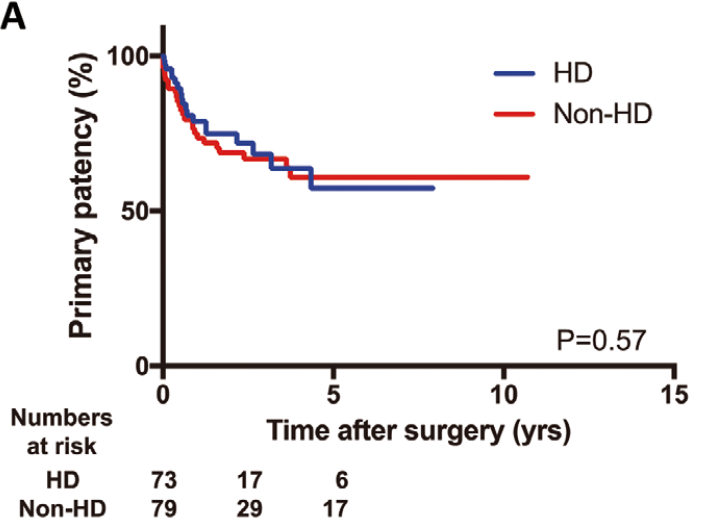

B

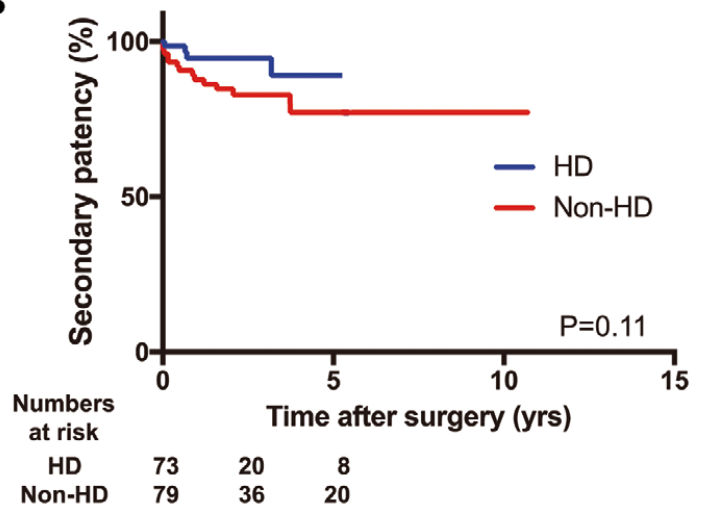

Figure 5. Long-term outcomes linked to the distal bypass procedure for critical limb ischemia: hemodialysis (HD) group vs. nonHD group. (A) Primary patency rate, (B) secondary patency rate.

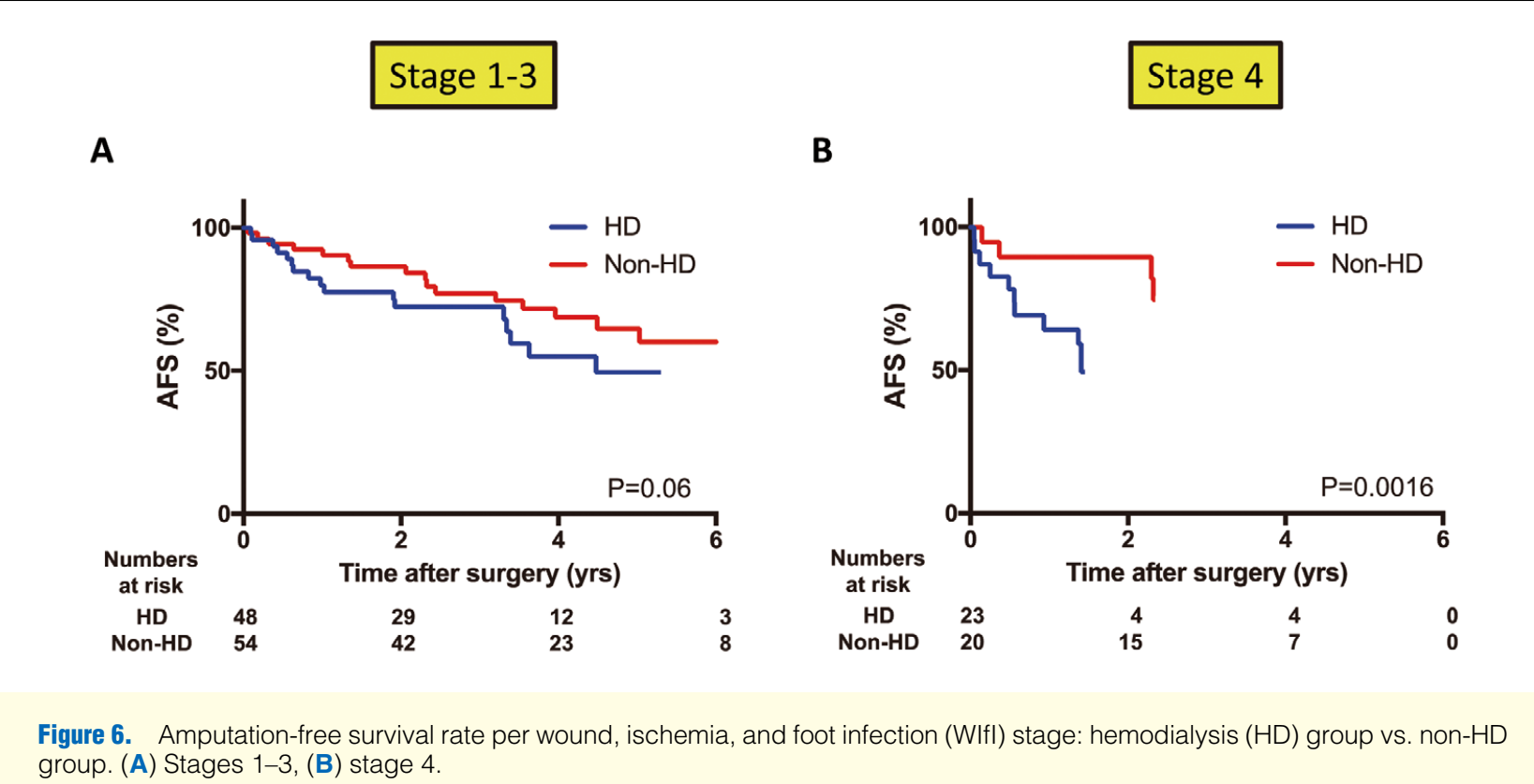

during distal bypass, it is clinically meaningful to compare the HD and non-HD groups.

In Japan, the rate of HD is obviously high. Even considering publication bias, the rate of HD patients in high-volume centers is high at $43-78 \%,,^{7-10,21}$ in contrast to rates of $10-14 \%$ in the USA and Europe. ${ }^{3,4,22}$ This might be related to differences in medical circumstances; it should be easy to introduce HD into the Japanese medical service system. In their treatment of patients with unique profiles, vascular surgeons have polished the surgical techniques and settled the procedural steps for HD-specific vessel conditions such as severe calcification. In the same way, multidisciplinary treatment, including foot care for DM, has spread worldwide, and the emergence of the WIfI classification makes it easy to evaluate foot conditions not only by doctors but also by medical staff, which might lead to improved limb prognosis.

As most of the limbs in the present study had tissue loss
(79\%), classical classifications such as Fontaine's or Rutherford's were not appropriate for predicting outcome, which was the reason we used the WIfI classification. Although a few short-term outcomes such as major amputation risk, AFS, and wound healing time are reported to be differentiated per WIfI stage, ${ }^{23}$ the evaluation of this new concept has not been established. ${ }^{24}$ By accumulating data, including images and clinical information collected from both doctors and medical staff, we were able to evaluate the patients' foot conditions with the WIfI classification. Thus we found that the AFS rate of the HD group was significantly worse in stage 4 compared with other stages. Considering the inherent operative risk in stage 4 patients with $\mathrm{HD}$, the surgical indication of stage 4 patients might need to be reconsidered, even if distal bypass itself could be performed in theory. Although we could not prove the down-staging effect of preoperative multidisciplinary treatment in this retrospective study, it is certain that we had some down-staged 
patients under our preoperative strategy. We hope that multidisciplinary treatment for CLI will prevail in the future and improve the limb outcomes. ${ }^{25}$

We reconfirmed the worse life prognosis of the HD group using the overall survival rate and MACE-free survival rate. The AFS rate, which was closely related to limb prognosis, was also worse in the HD group. This trend was the same as seen in our previous study. ${ }^{7}$ Considering the poor life prognosis of the HD group, we think the limb salvage rate does not necessarily reflect the real limb prognosis. Instead, the primary and secondary patency rates would better reflect the procedural outcomes. As there was no difference in patency between the groups, we reconfirmed that distal bypass for patients on HD should still be the first-line treatment.

\section{Study Limitations}

First, there are confounders in comparing the 2 groups, and the number of patients was too small for matching of the groups. Next, this study was based on retrospective data from a single institution, where a selection bias exists. Another limitation was that there might have been a selection bias for the surgical indication, which should be limited by simply comparing the $\mathrm{HD}$ and non-HD groups. Nevertheless, this study revealed the real-world outcomes of CLI patients with HD after distal bypass surgery.

\section{Conclusions}

The life and limb prognoses of CLI patients with HD were worse than those of non-HD patients, as indicated by the overall survival, MACE-free survival and AFS rates. There was no difference in surgical outcomes suggested by the primary and secondary graft patency rates between the 2 groups. AFS in WIfI stage 4 was significantly worse in the HD group, which indicates the importance of the preoperative limb status.

\section{Acknowledgments}

None.

\section{Conflict of Interest}

There is no conflict of interest to declare.

\section{References}

1. Bradbury AW, Adam DJ, Bell J, Forbes JF, Fowkes GR, Gillespie I, et al. Bypass versus Angioplasty in Severe Ischaemia of the Leg (BASIL) trial: Analysis of amputation free and overall survival by treatment received. J Vasc Surg 2010; 51: 18 S-31S.

2. Bradbury AW, Adam DJ, Bell J, Forbes JF, Fowkes GR, Gillespie I, et al. Bypass versus Angioplasty in Severe Ischaemia of the Leg (BASIL) trial: An intention-to-treat analysis of amputation-free and overall survival in patients randomized to a bypass surgery-first or a balloon angioplasty-first revascularization strategy. J Vasc Surg 2010; 51: 5S-17S.

3. Nolan BW, De Martino RR, Stone DH, Schanzer A, Goodney PP, Walsh DW, et al. Prior failed ipsilateral percutaneous endovascular intervention in patients with critical limb ischemia predicts poor outcome after lower extremity bypass. J Vasc Surg 2011; 54: $730-736$.

4. Reinecke H, Unrath M, Freisinger E, Bunzemeier H, Meyborg M, Luders F, et al. Peripheral arterial disease and critical limb ischaemia: Still poor outcomes and lack of guideline adherence. Eur Heart J 2015; 36: 932-938.

5. Norgren L, Hiatt WR, Dormandy JA, Nehler MR, Harris KA, Fowkes FG, et al. Inter-society consensus for the management of peripheral arterial disease (TASC II). J Vasc Surg 2007; 45(Suppl S):
S5-S67.

6. Masakane I, Nakai S, Ogata S, Kimata N, Hanafusa N, Hamano T, et al. An overview of regular dialysis treatment in Japan (As of 31 December 2013). Ther Apher Dial 2015; 19: 540-574.

7. Yamamoto S, Hosaka A, Okamoto H, Shigematsu K, Miyata T, Watanabe T. Efficacy of revascularization for critical limb ischemia in patients with end-stage renal disease. Eur J Vasc Endovasc Surg 2014; 48: 316-324.

8. Kodama A, Sugimoto M, Kuma S, Okazaki J, Mii S, Komori K. Clinical outcomes after infrainguinal bypass grafting for critical limb ischemia in patients with dialysis-dependent end-stage renal failure. Eur J Vasc Endovasc Surg 2014; 48: 695-702.

9. Nakano M, Hirano K, Iida O, Soga Y, Kawasaki D, Suzuki K, et al. Prognosis of critical limb ischemia in hemodialysis patients after isolated infrapopliteal balloon angioplasty: Results from the Japan Below-the-Knee Artery Treatment (J-BEAT) Registry. J Endovasc Surg 2013; 20: 113-124.

10. Azuma N, Uchida H, Kokubo T, Koya A, Akasaka N, Sasajima T. Factors influencing wound healing of critical ischaemic foot after bypass surgery: Is the angiosome important in selecting bypass target artery? Eur J Vasc Endovasc Surg 2012; 43: 322-328.

11. Miyahara T, Suhara M, Nemoto Y, Shirasu T, Haga M, Mochizuki $\mathrm{Y}$, et al. Long-term results of treatment for critical limb ischemia. Ann Vasc Dis 2015; 8: 192-197.

12. Mills JL Sr, Conte MS, Armstrong DG, Pomposelli FB, Schanzer A, Sidawy AN, et al. The society for vascular surgery lower extremity threatened limb classification system: Risk stratification based on wound, ischemia, and foot infection (WIfI). J Vasc Surg 2014; 59: 220-234.e1-e2

13. Shirasu T, Hoshina K, Nishiyama A, Akagi D, Miyahara T, Yamamoto $\mathrm{K}$, et al. Favorable outcomes of very elderly patients with critical limb ischemia who undergo distal bypass surgery. J Vasc Surg 2016; 63: 377-384.

14. Urabe G, Yamamoto K, Onozuka A, Miyata T, Nagawa H. Skin perfusion pressure is a useful tool for evaluating outcome of ischemic foot ulcers with conservative therapy. Ann Vasc Dis 2009; 2: $21-26$.

15. Mochizuki Y, Hoshina K, Shigematsu K, Miyata T, Watanabe T. Distal bypass to a critically ischemic foot increases the skin perfusion pressure at the opposite site of the distal anastomosis. Vascular 2016; 24: 361-367.

16. Mochizuki Y, Hosaka A, Kamiuchi H, Nie JX, Masamune K, Hoshina K, et al. New simple image overlay system using a tablet PC for pinpoint identification of the appropriate site for anastomosis in peripheral arterial reconstruction. Surg Today 2016 March 17, doi:10.1007/s00595-016-1326-4.

17. Conte MS. Critical appraisal of surgical revascularization for critical limb ischemia. J Vasc Surg 2013; 57: 8S-13S.

18. Schiffrin EL, Lipman ML, Mann JF. Chronic kidney disease: Effects on the cardiovascular system. Circulation 2007; 116: 85-97.

19. Reslerova M, Moe SM. Vascular calcification in dialysis patients: Pathogenesis and consequences. Am J Kidney Dis 2003; 41: S96S99.

20. Moe SM, Chen NX. Pathophysiology of vascular calcification in chronic kidney disease. Circ Res 2004; 95: 560-567.

21. Kumada Y, Nogaki H, Ishii H, Aoyama T, Kamoi D, Takahashi H, et al. Clinical outcome after infrapopliteal bypass surgery in chronic hemodialysis patients with critical limb ischemia. J Vasc Surg 2015; 61: 400-404.

22. Patel VI, Mukhopadhyay S, Guest JM, Conrad MF, Watkins MT, Kwolek CJ, et al. Impact of severe chronic kidney disease on outcomes of infrainguinal peripheral arterial intervention. J Vasc Surg 2014; 59: $368-375$.

23. Zhan LX, Branco BC, Armstrong DG, Mills JL Sr. The Society for Vascular Surgery lower extremity threatened limb classification system based on Wound, Ischemia, and for Infection (WIfI) correlates with risk of major amputation and time to wound healing. $J$ Vasc Surg 2015; 61: 939-944.

24. Azuma N, Koya A, Uchida D, Saito Y, Uchida H. Ulcer healing after peripheral intervention-can we predict it before revascularization? Circ J 2014; 78: 1791 - 1800 .

25. Kawarada O, Yasuda S, Huang J, Honda Y, Fitzgerald PJ, Ishihara $\mathrm{M}$, Ogawa H. Contemporary infrapopliteal intervention for limb salvage and wound healing: Harmonization of revascularization and wound management. Circ J 2014; 78: 1540-1549. 